\title{
Mineral-enriched deep-sea water inhibits the metastatic potential of human breast cancer cell lines
}

\author{
SOYOUNG KIM, SO-YOUNG CHUN, DO-HYUNG LEE, KYU-SHIK LEE and KYUNG-SOO NAM \\ Department of Pharmacology, College of Medicine and Intractable Disease Research Center, \\ Dongguk University, Gyeongju 780-714, Republic of Korea
}

Received July 2, 2013; Accepted August 19, 2013

DOI: $10.3892 /$ ijo.2013.2089

\begin{abstract}
Recently, the scientific community has begun to establish the health benefits of deep-sea water (DSW) due to its enrichment in nutrients and minerals. In this study, we investigated the effects of deep-sea water (DSW) on the metastatic potential of two human breast cancer cell lines exhibiting highly different phenotypes. MDA-MB-231 cells exhibit invasive/metastatic tumor features with rapid migration ability and high endogenous expression of TGF- $\beta$ and Wnt5a. DSW treatment significantly inhibits their migratory ability in a wound-healing assay. This inhibitory effect of DSW appears to be mediated through TGF- $\beta$ and Wnt5a signaling, resulting in attenuated expression of CD44. We further investigated the preventive effect of DSW on 12- $O$-tetradecanoylphorbol-13-acetate (TPA)-induced invasive/metastatic tumor features in non-invasive MCF-7 cells. Similar to the inhibitory effects shown in MDA-MB-231 cells, we observed that DSW treatment resulted in the inhibition of TPA-induced migration and MMP-9 activity with a concomitant decrease in mRNA levels of MMP-9, TGF- $\beta$, Wnt5a and Wnt3a. Taken together, our data show that DSW has inhibitory effects on breast cancer invasion/metastasis, suggesting that DSW has some promise in improving cancer survival by preventing tumor metastasis.
\end{abstract}

Correspondence to: Professor Kyung-Soo Nam, Department of Pharmacology, College of Medicine and Intractable Disease Research Center, Dongguk University, Gyeongju 780-714, Republic of Korea

E-mail:namks@dongguk.ac.kr

Abbreviations: DSW, deep-sea water; TPA, 12-O-tetradecanoylphorbol-13-acetate; COX-2, cyclooxygenase-2; TGF- $\beta$, transforming growth factor- $\beta$; uPA, urokinase plasminogen activator; uPAR, urokinase plasminogen activator receptor; ER, estrogen receptor; PR, progesterone receptor; FZD, Frizzled; LRP5/6, low-density lipoprotein receptors $5 / 6$

Key words: deep-sea water, human breast cancer cells, metastasis, matrix metalloproteinase- 9 , transforming growth factor- $\beta$, Wnt5a

\section{Introduction}

Deep-sea water (DSW) defined as sea water from a depth of more than 200 meters is rich in minerals such as calcium $(\mathrm{Ca})$, magnesium $(\mathrm{Mg})$, potassium $(\mathrm{K})$, sodium $(\mathrm{Na})$, and zinc $(\mathrm{Zn})$. Hence, DSW has been widely utilized in the fields of aquaculture, agriculture, food processing and cosmetics (1). More recently, the scientific community has begun to establish the health benefits of DSW which include lowering of blood cholesterol and preventing obesity and diabetics (2-4). However, these studies are preliminary yet and applications of DSW in medical fields require more scientific evidence for its biological activities. Previously, we showed the inhibitory effects of DSW on carcinogen-induced expression of cyclooxygenase-2 (COX-2), transforming growth factor- $\beta$ (TGF- $\beta$ ), and urokinase plasminogen activator (UPA) in HT-29 colorectal cancer cells (5). It is presumed that these antitumor activities of DSW may be derived from the combined ionic action of several minerals such as calcium, magnesium and potassium in DSW. Among these minerals, calcium and magnesium may play important roles in mediating the inhibition of metastasis because they are the main mineral ions present in DSW and it was found that deficiency of magnesium and/or calcium levels has been linked to increased risks of cancer and metastasis (6-10).

Metastasis is a major cause of lethality in cancer patients rather than the primary tumors. Recurrent metastatic disease is estimated to develop in $30-75 \%$ of patients undergoing surgery and adjuvant treatment. The median survival of patients with metastatic breast cancer is about 2 years after metastasis has been detected (11). Thus, understanding the mechanism involved in the regulation of metastasis is of major importance for improving cancer survival. The mechanism underlying tumor metastasis remains unclear but considerable focus has been directed towards characterizing metastasis genes in the context of relevant signaling pathways.

Several studies have demonstrated the implication of TGF- $\beta$ signaling in cell invasion and metastasis. Although TGF- $\beta$ has a complex role in tumor progression by acting as a tumor suppressor or tumor promoter depending on the tumor type and the tumor stage, the clinical and experimental evidence implies the involvement of TGF- $\beta$ in the metastatic processes (12). Moreover, elevated levels of plasma TGF- $\beta$ have been detected in patients with cancer and predicts early metastasis $(13,14)$. It has been shown that activation of TGF- $\beta$ signaling accelerates 
tumor growth, migration and metastasis whereas blockade of TGF- $\beta$ signaling reduces the development of bone metastasis in animal models $(15,16)$.

Recent studies identified that alterations in TGF- $\beta$ signaling regulated Wingless-related mouse mammary tumor virus (MMTV) integration site 5A (Wnt5a) gene expression. Wnt5a is a non-canonical signaling member of the Wnt family, activation of which is independent of $\beta$-catenin. In contrast, Wnt1 and Wnt3a are canonical Wnt signaling members, that bind to Wnt receptors such as Frizzled (FZD) and low-density lipoprotein receptors 5/6 (LRP5/6) to promote stabilization and nuclear translocation of $\beta$-catenin, resulting in the activation of target genes (17). It is found that TGF- $\beta$ directly upregulated Wnt5a expression through the Smad complex and the Wnt5a promoter reserved Smad binding sites (18). Wnt5a is also associated with poor prognosis and early invasive breast cancer, suggesting the involvement of Wnt5a in malignant transformation and tumor progression $(19,20)$. Its overexpression is observed in a variety of cancers in comparison to the respective benign tissues. Moreover, a study analyzing gene expression profiles with cutaneous melanomas revealed that Wnt5a expression was one of the most robust markers for highly aggressive tumors, while it was underexpressed in the less motile tumors (21). Transfection of Wnt5a in non-invasive melanoma cells expressing low levels of Wnt5a resulted in increased invasiveness via PKC activation (22). Although a role for Wnt5a in mediating motility is not entirely clear, it is found that the treatment with recombinant Wnt5a upregulates expression of CD44 (23), which is a tumor homing and metastasis antigen associated with tumor cell invasion (24).

Expression of a gene other than CD44 is also involved in Wnt5a-dependent invasion and metastasis. Recently, binding of Wnt5a to Ror2, a member of the Ror-family of receptor-tyrosine kinases, was reported to regulate the expression of matrix metalloproteinases (MMPs) (25). MMPs belong to a family of zinc-dependent enzymes capable of degrading extracellular matrix, and thus mediate the physiological processes involving extracellular matrix remodeling, such as wound healing, angiogenesis and tumor progression (26). Direct evidence for involvement of MMPs in tumor invasion was revealed through studies in which MMP-9 knockout mice had reduced melanoma tumor progression and angiogenesis (27).

In this study, we have evaluated the potential health benefits of DSW on the inhibition of the metastatic potential in relation to tumor cell migration, TGF- $\beta$ and its relevant signaling pathway, such as Wnt5a, CD44 and MMPs, by using two human breast cancer cell lines (MDA-MB-231 and MCF-7). MDA-MB-231 cells are representative of triple negative breast tumor characterized by the absence of estrogen receptor (ER), progesterone receptor (PR), and Her2. Recent studies analyzing gene expression profiles reveal that the triple negative tumor subtypes highly express genes regulating tumor migration, invasion, and differentiation, including the TGF- $\beta$ signaling pathway and the Wnt-signaling pathway $(28,29)$. This triple negative tumor subtype is mainly correlated to poor outcomes, showing the worst overall and disease-free survival rates due to its metastatic and invasive features. MDA-MB-231 cells exhibited invasive/metastatic tumor features with rapid migratory ability and relatively high endogenous expression of TGF- $\beta$ and Wnt5a (30). In contrast to MDA-MB-231 cells,
MCF-7 cells are non-invasive ER-positive breast cancer cells, representing the ER/PR positive luminal subtype. Despite the fact that ER/PR positive luminal subtypes have been found to be associated with the most favorable outcomes, tumor recurrent disease in patients undergoing chemotherapy exhibited metastatic tumor features. Thus, we used MDA-MB-231 cells to evaluate whether DSW directly inhibits the invasive behavior of tumor features in breast cancer cells. Besides, the non-invasive MCF-7 cells were used with treatment of 12-O-tetradecanoylphorbol-13-acetate (TPA) to examine the preventive effects of DSW on TPA-induced invasive/metastatic tumor characteristics.

\section{Materials and methods}

Preparation of deep-sea water. Marine Deep Ocean Water Application Center in Korea Institute of Ocean Science and Technology (Goseong, Korea) provided desalinated water and deep-sea water of hardness 4,000. The ratio of magnesium to calcium was 3:1 and the hardness of DSW was determined from the concentration of calcium and magnesium ions within DSW. The following equation was used to calculate the hardness of DSW in this study: Hardness (mg/l) = magnesium $(\mathrm{mg} / \mathrm{l}) \times 4.1+$ calcium $(\mathrm{mg} / \mathrm{l}) \times 2.5$.

To prepare DSW containing media, DMEM powder was dissolved in hardness 4,000 DSW and diluted with distilled water to obtain hardness $1,500 \mathrm{DSW}$ media. It was further serially diluted with desalinated media to prepare hardness 200-800 media.

Cell culture. MCF-7 and MDA-MB-231 human breast cancer cell lines were purchased from the Korean Cell Line Bank (Seoul, Korea). MCF-7 cells were cultured in DMEM (Gibco-BRL, Rockville, MD, USA) supplemented with $10 \%$ fetal bovine serum and $10 \mu \mathrm{g} / \mathrm{ml}$ insulin, while MDA-MB231 cells were cultured in DMEM supplemented with $10 \%$ fetal bovine serum without insulin.

RNA isolation and reverse transcriptase polymerase chain reaction ( $R T-P C R)$. MCF-7 cells were seeded on 6-well plates and cultured for $24 \mathrm{~h}$. After $24 \mathrm{~h}$ serum starvation with serum-free media, cells were treated with conditioned media containing various hardness of DSW for $2 \mathrm{~h}$ prior to adding 100 nM TPA (Sigma, St. Louis, MO, USA). Cells were further incubated for $24 \mathrm{~h}$ and harvested for RNA isolation. For treatment of MDA-MB-231 cells with DSW, cells were cultured for 3 days in the presence of conditioned media containing various hardness of DSW and $2 \%$ fetal bovine serum and harvested for RNA isolation. Total RNA was isolated using easy-BLUE ${ }^{\mathrm{TM}}$ Total RNA Extraction kit (iNtRON Biotechnology Inc., Sungnam, Korea). cDNA synthesis and PCR reactions were performed as previously described (5). Primer sequences and PCR conditions for target genes are presented in Table I. Densitometric analysis was performed using Scion Image (Scion Corporation, Frederick, MD, USA).

Wound healing assay. MCF-7 cells were seeded on 6-well plates coated with collagen IV and grown to reach confluence. Monolayers of cells were scratched with a $200 \mu$ l yellow pipette tip to create wounds. After washing cells with PBS to 
Table I. Primer sequences and annealing temperatures for RT-PCR.

\begin{tabular}{|c|c|c|c|}
\hline Gene & Primer & $\begin{array}{c}\text { Annealing } \\
\text { temperature } \\
\left({ }^{\circ} \mathrm{C}\right)\end{array}$ & Authors/(Refs.) \\
\hline MMP-9 & $\begin{array}{l}\text { Forward: 5'-TTCATCTTCCAAGGCCAATC-3' } \\
\text { Reverse: 5'-CTTGTCGCTGTCAAAGTTCG-3' }\end{array}$ & 50 & Jang et al (45) \\
\hline MMP-2 & $\begin{array}{l}\text { Forward: 5'-TCGCCCATCATCAAGTTC-3' } \\
\text { Reverse: 5'-GTGATCTGGTTCTTGTCC-3' }\end{array}$ & 52 & Kang et al (46) \\
\hline TGF- $\beta$ & $\begin{array}{l}\text { Forward: 5'-CGTCTGCTGAGGAGGCTCAAGTTA-3' } \\
\text { Reverse: 5'-CAGCCGAGGTCCTTGCGGAA-3' }\end{array}$ & 55 & \\
\hline uPA & $\begin{array}{l}\text { Forward: 5'-CCAATTAGGAAGTGTAACAGC-3' } \\
\text { Reverse: } \\
\text { 5'-GCCAAGAAAGGGACATCTATG-3' }\end{array}$ & 55 & Kang et al (46) \\
\hline UPAR & $\begin{array}{l}\text { Forward: 5'-CACAAAACTGCCTCCTTCCTA-3' } \\
\text { Reverse: 5'-AATCCCCGTTGGTCTTACAC-3' }\end{array}$ & 58 & Li and Sarkar (47) \\
\hline Wnt5a & $\begin{array}{l}\text { Forward: 5'-GGGAGGTTGGCTTGAACATG-3' } \\
\text { Reverse: 5'-GAATGGCACGCAATTACCTT-3' }\end{array}$ & 58 & Suzuki et al (48) \\
\hline Wnt3a & $\begin{array}{l}\text { Forward: 5'-TGAACAAGCACAACAACGAG-3' } \\
\text { Reverse: } 5 \text { '-CAGTGGCATTTTTCCTTCC-3' }\end{array}$ & 55 & Suzuki et al (48) \\
\hline$\beta$-actin & $\begin{array}{l}\text { Forward: 5'-CAAGAGATGGCCACGGCTGCT-3' } \\
\text { Reverse: 5'-TCCTTCTGCATCCTGTCGGCA-3' }\end{array}$ & 58 & Takeuchi et al (49) \\
\hline GAPDH & $\begin{array}{l}\text { Forward: 5'-ATCCCATCACCATCTTCCAG-3' } \\
\text { Reverse: } 5 \text { '-TTCTAGACGGCAGGTCAGGT-3' }\end{array}$ & 58 & \\
\hline
\end{tabular}

remove cell debris, cells were treated with conditioned media containing various hardness of DSW for $1 \mathrm{~h}$ prior to addition of $100 \mathrm{nM}$ TPA, and pictures were taken at time 0 , and at indicated time points. The wound healing assay for MDA-MB-231 cells was performed as described above, but stimulation of migration by $100 \mathrm{nM}$ TPA was omitted due to the inherent invasive properties of the cells.

Gelatin zymography assay. Conditioned medium was electrophoresed in an $8 \%$ sodium dodecyl sulfate (SDS)polyacrylamide gel containing $0.1 \%(\mathrm{v} / \mathrm{v})$ gelatin. The gel was then washed at room temperature for $1 \mathrm{~h}$ with $0.25 \%$ Triton X-100 and subsequently incubated at room temperature for $24 \mathrm{~h}$ in reaction buffer containing $5 \mathrm{mM} \mathrm{CaCl}_{2}, 0.04 \%$ $\mathrm{NaN}_{3}$ and $50 \mathrm{mM}$ Tris-HCl. The gel was stained with $0.2 \%$ Coomassie Brilliant Blue and proteolysis was detected as a white zone in a dark blue field.

Flow cytometry analysis. Cells were trypsinized and washed in $2 \%$ FBS in PBS. Cells were incubated with CD44-FITC (BD Biosciences, San Diego, CA, USA) for $30 \mathrm{~min}$ on ice and washed with $2 \%$ FBS in PBS. Cells were resuspended in a final volume of $500 \mu \mathrm{l}$ buffer for analysis. FACS analysis was performed on a FACSCalibur II (BD Biosciences). Unstained and single color-labeled samples were used to calibrate the analyzer for each experiment.

\section{Results}

Effect of DSW on MDA-MB-231 invasive human breast cancer cells. Since MDA-MB-231 cells provide a model of aggressive human breast cancer, we tested whether DSW inhibits their migratory ability in an in vitro wound healing assay. MDA-MB-231 cells showed rapid wound closure at all time points, but treatment of cells with different hardness of DSW for up to 2 days significantly reduced migration of MDA-MB-231 cells in a dose-dependent manner (Fig. 1A). The role of MMPs in cancer invasion and metastasis is crucial due to their ability to degrade extracellular matrix proteins, which is an essential process for cancer invasion and metastasis that allows the cancer cells to be released from site of the primary tumor and to penetrate to the nearby tissues or spread to other parts of the body (26). Therefore, we evaluated the effects of DSW on the expression of MMPs. MMP-2 is usually expressed constitutively, while the synthesis and secretion of MMP-9 are induced by a variety of stimuli including cytokines and TPA. Since the relative levels of MMP-2 are higher in the highly invasive and metastatic MDA-MB-231 cell line, we evaluated the inhibitory effect of DSW on both the endogenous gene expression of MMP-2 of MDA-MB-231 cells and TPA-induced MMP-9 gene expression. Although our data suggested that DSW had mild inhibitory effects on the MMP-2 gene expression, exhibiting approximately $20 \%$ of inhibition at 1,500 hardness (Fig. 1B), TPA-induced MMP-9 expression was significantly inhibited by the treatment with DSW (Fig. 1C). The endogenous level of MMP-9 gene expression was barely detectable in MDA-MB-231 cells, while treatment with TPA for $24 \mathrm{~h}$ induced MMP-9 gene expression by about 3-fold. However, treatment with DSW showed clear inhibitory effects on MMP-9 gene expression; in particular, cells treated with conditioned media containing 1,500 hardness DSW showed almost basal level expression of MMP-9. Therefore, our data suggested that DSW may have inhibitory effects on the mechanisms underlying cancer invasion and metastasis. 
A
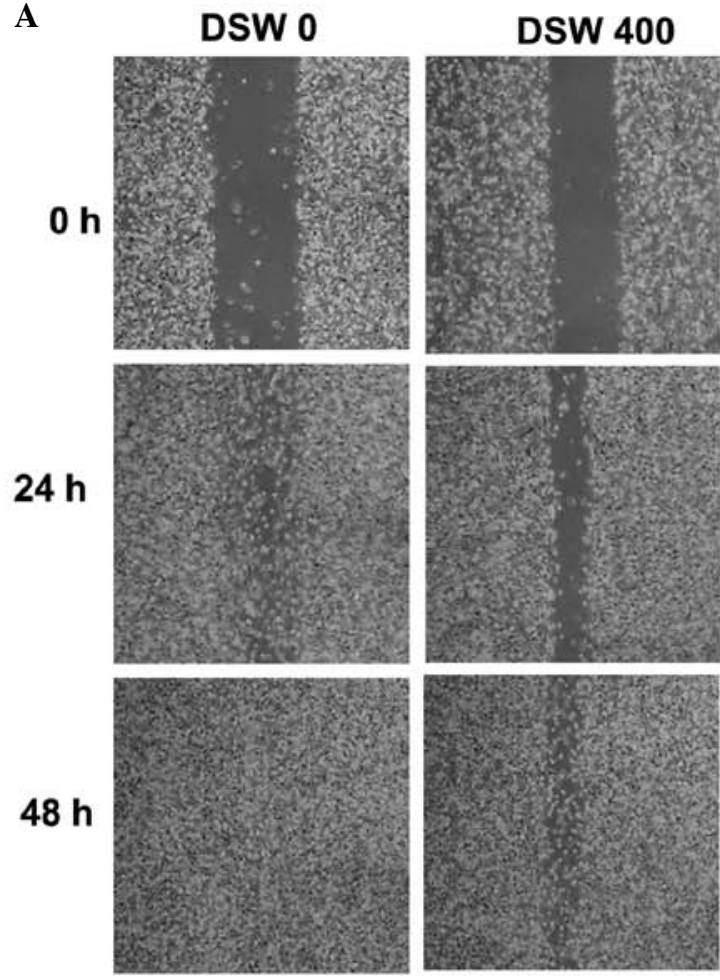

B
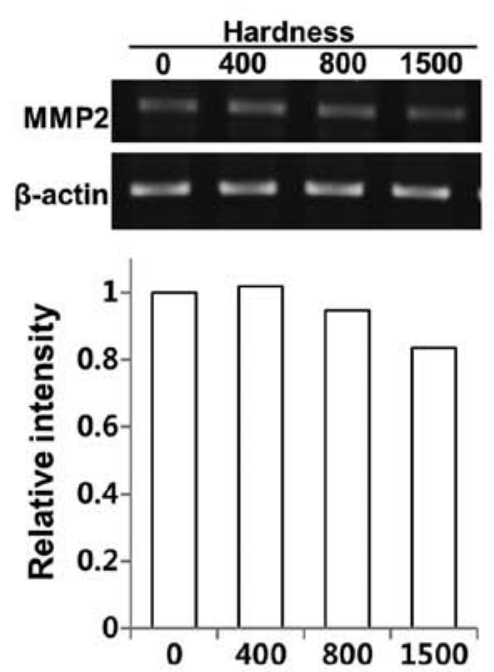

DSW 400

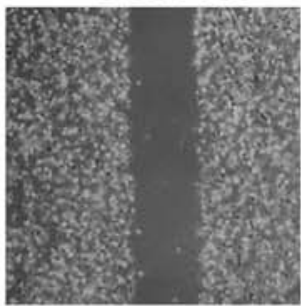

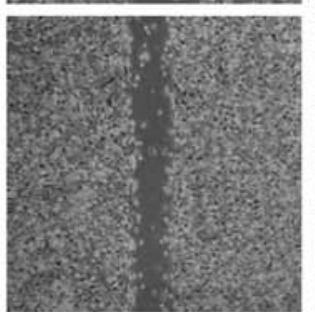
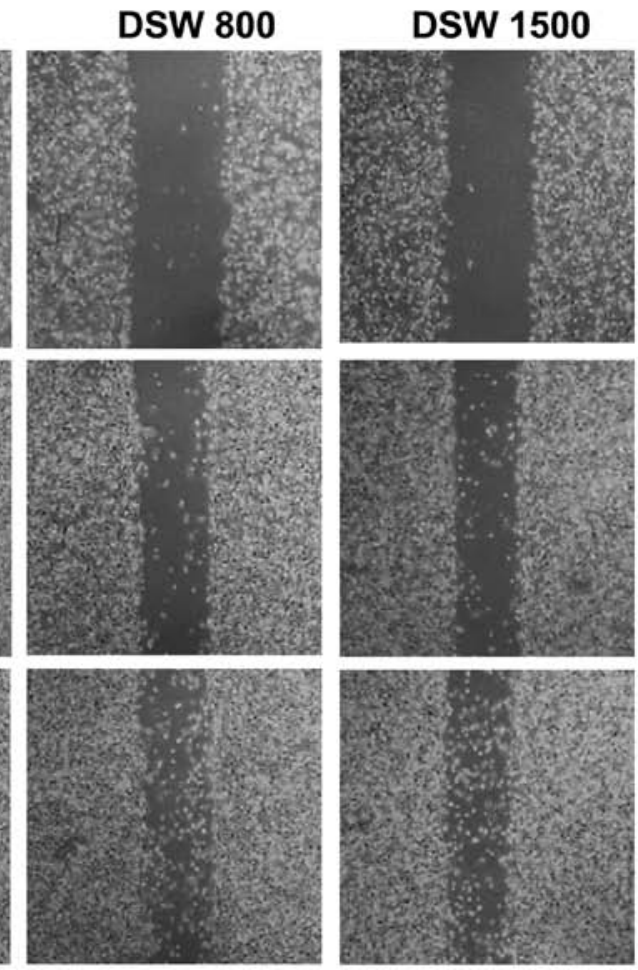

C
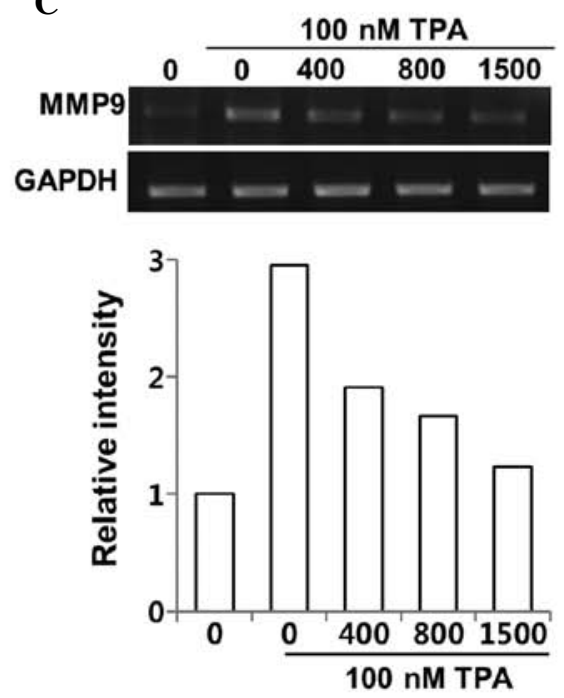

Figure 1. Inhibitory effect of DSW on migratory ability of invasive MDA-MB-231 cells. (A) Optical microscopic images of in vitro wound healing at 0,24 and $48 \mathrm{~h}$ after creation of wounds. MDA-MB-231 cells showed rapid wound closure at all time points, but treatment of cells with conditioned media containing various hardness of DSW from 400-1,500 for up to 2 days results in a decrease in the ability of cells to close the wound in the wound healing assay. Effects of DSW on mRNA expression of (B) MMP-2 and (C) MMP-9. For evaluation of DSW effect on MMP-9 gene expression, MMP-9 expression was induced by 24 h treatment with TPA in cells pretreated with different hardness of DSW for $2 \mathrm{~h}$. The relative intensity of each sample was expressed as fold change compared to control after normalization to a respective loading control.

Regulation of the expression of TGF- $\beta$ and its direct mediator, Wnt5a, by DSW in MDA-MB-231 cells. Cell migration and invasion are complex requiring coordination of many signaling pathways. We first evaluated whether the inhibitory effects of DSW on migratory ability of MDA-MB-231 cells occurred from altered TGF- $\beta$ signaling upon treatment with DSW. As shown in Fig. 2A, treatment with DSW for 3 days inhibited the TGF- $\beta$ gene expression of MDA-MB-231 cells; the cells treated with the conditioned media containing 800 or
1,500 hardness DSW exhibited about 40\% inhibition (Fig. 2A). The expression of Wnt5a, a direct mediator of TGF- $\beta$, was also affected by DSW (Fig. 2B) but the inhibitory effect of DSW on Wnt5a gene expression was relatively mild compared to its effect on the TGF- $\beta$ gene expression.

Attenuated CD44 expression in MDA-MB-231 cells cultured in $D M E M$ with different hardness of DSW. Wnt5a overexpression or knockdown using siRNA identified CD44 as one of the Wnt5a 

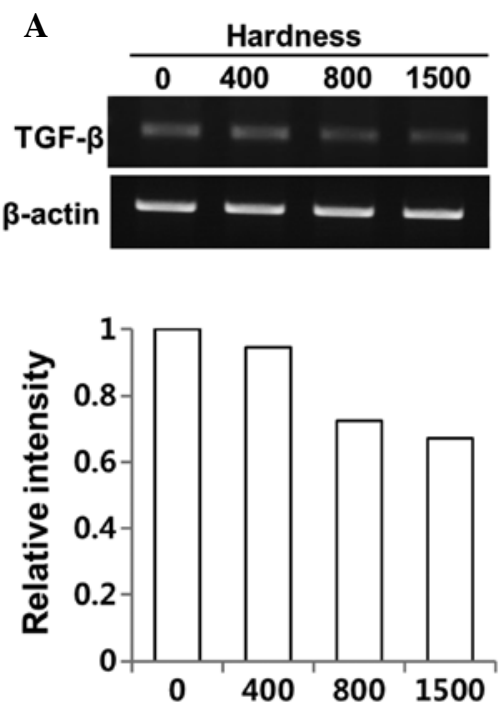

B
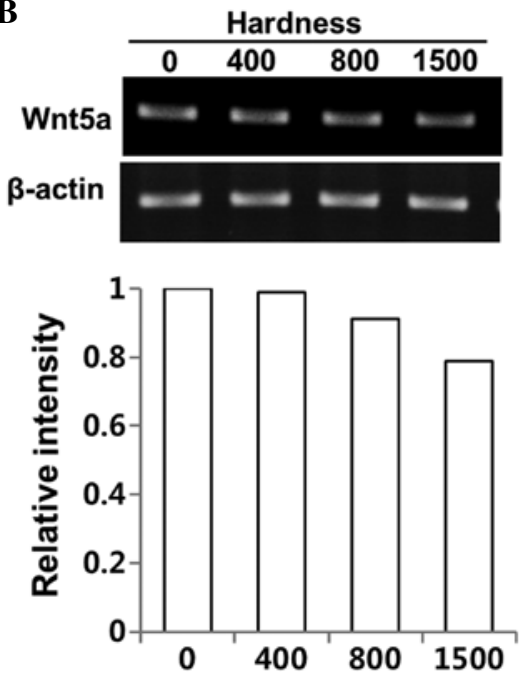

Figure 2. Regulation of TGF- $\beta$ and Wnt5a expression by DSW in MDA-MB-231 cells. MDA-MB-231 cells were cultured for 3 days in the presence of conditioned media containing various hardness of DSW and $2 \%$ fetal bovine serum and harvested for RNA isolation. RT-PCR was performed for (A) TGF- $\beta$ and (B) Wnt5a, and their relative intensity in each sample was expressed as fold change compared to control after normalization to a respective loading control.

target genes (23). Studies analyzing gene expression profiles with 52 breast cancer cell lines revealed that CD44 amplification is correlated to triple negative breast cancer cell lines including MDA-MB-231 cells (31). Since CD44 is identified as a Wnt5a target gene and is highly expressed in MDA-MB-231 cells, we tested whether DSW can affect the expression of CD44 in MDA-MB-231 cells. Flow cytometric analysis was performed to measure CD44 expression in MDA-MB-231 cells treated with different hardness of DSW for 3 days. CD44 was highly expressed in MDA-MB-231 cells but treatment with DSW attenuated overall expression of CD44, suggesting that the inhibitory effect of DSW on Wnt5a expression subsequently affected the expression of CD44 in MDA-MB-231 cells (Fig. 3).
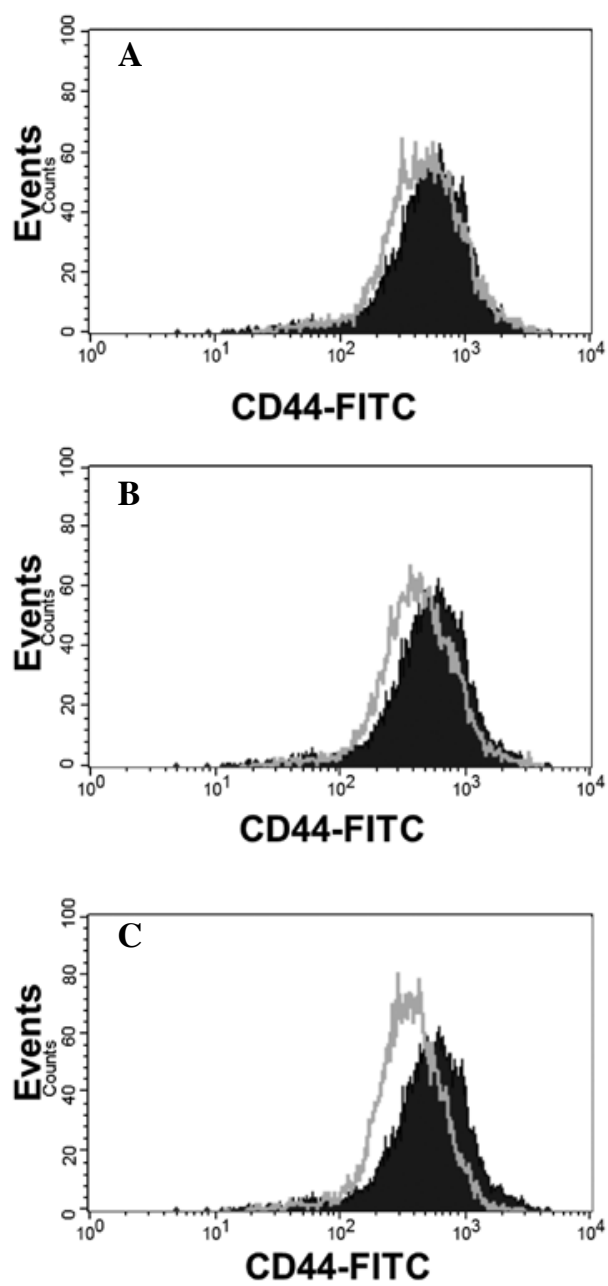

Figure 3. Flow cytometry analysis of CD44 expression in MDA-MB-231 cells treated with various hardness of DSW. CD44 expression was measured in MDA-MB-231 cells treated with different hardness of DSW for 3 days using flow cytometry and a FITC-conjugated CD44 antibody. Filled histogram represents endogenous expression of CD44 in untreated cells, while solid lines indicate CD44 expression in cells treated with (A) 400, (B) 800 and (C) 1,500 hardness DSW.

Effect of DSW on TPA-induced migration in non-invasive human breast cancer cells. To evaluate the preventive effect of DSW on TPA-induced invasive/metastatic tumor features, the migratory ability of TPA-treated MCF7 cells was evaluated using an in vitro wound healing assay. MCF-7 cells are weakly invasive in vitro, but treatment with TPA resulted in more rapid wound closure at all-time points (Fig. 4A). Treatment of the cells with different hardness of DSW for up to 2 days attenuated the TPA-induced migration in a dose-dependent manner compared to that in TPA-treated control cells (Fig. 4A). We further investigated the effects of DSW on the activity and expression of MMP-9 in MCF-7 cells. Since the endogenous level of MMP-2 is undetectable in non-invasive MCF-7 breast cancer cells, we evaluated the inhibitory effect of DSW on MMP-9 activity in a gelatin zymography assay after inducing the MMP-9 expression by treatment with TPA. The media from control cells showed barely detectable proteolytic activity, while treatment with TPA for $24 \mathrm{~h}$ induced MMP-9 secretion by about 18 -fold compared to control cells. However, the MMP-9 activity from cells treated with conditioned media 
A
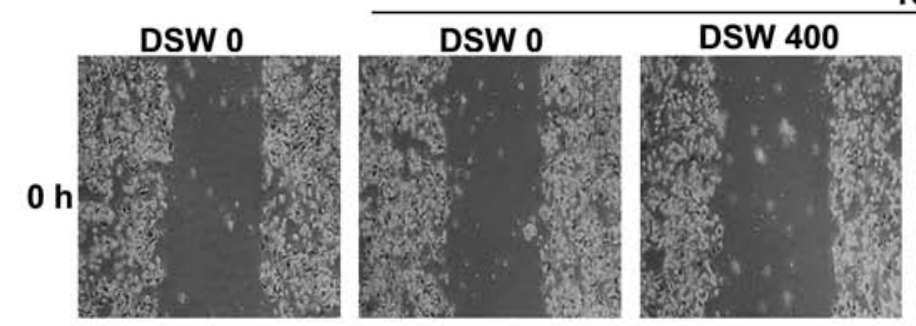

100 nM TPA
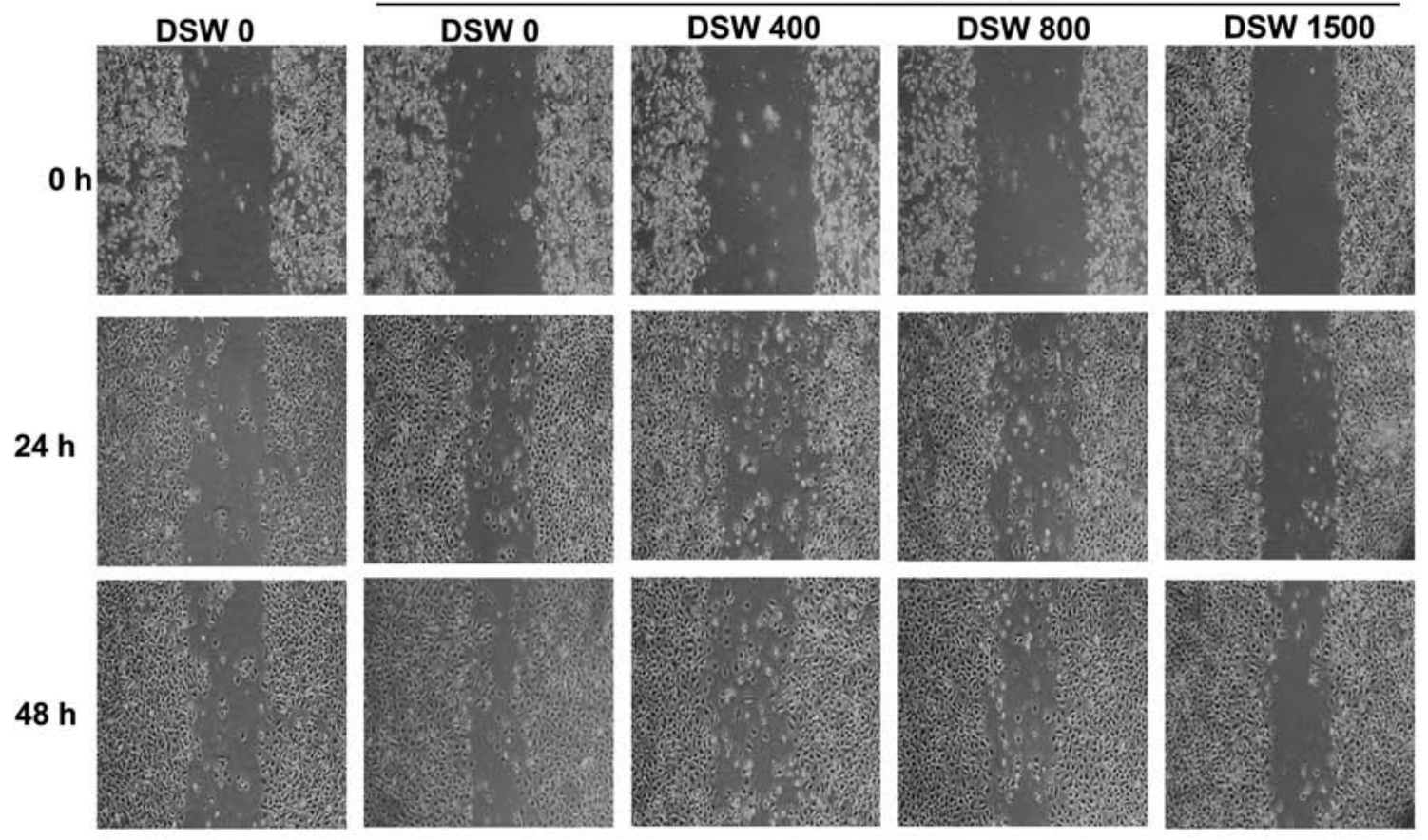

B
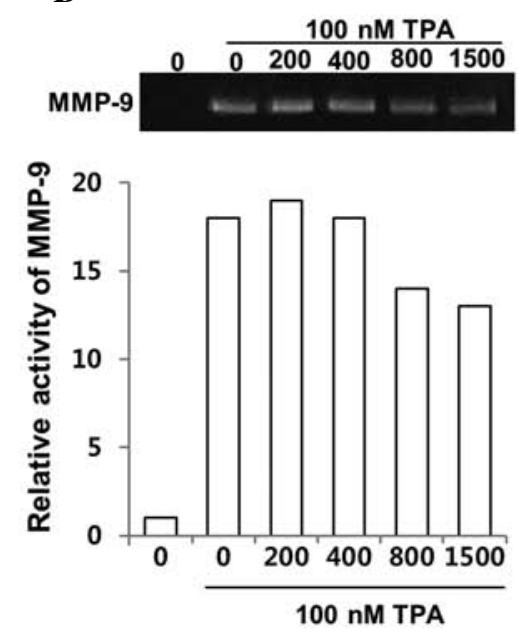
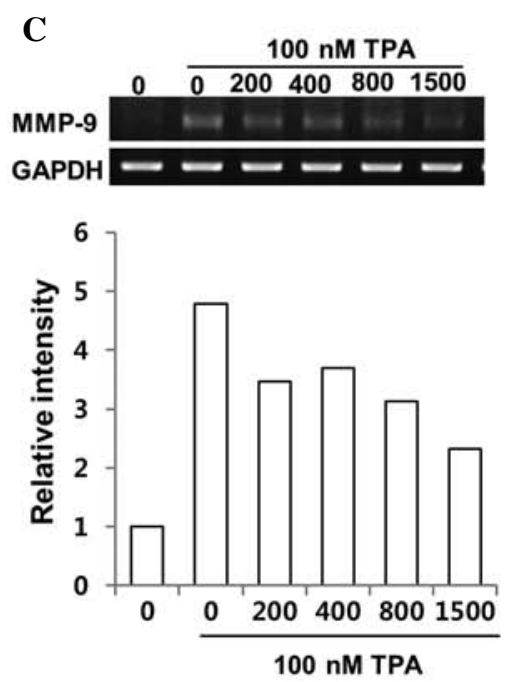

Figure 4. Inhibitory effects of DSW on migratory ability and MMP-9 activity of TPA-treated MCF-7 cells. (A) Optical microscopic images of in vitro wound healing at 0,24 and $48 \mathrm{~h}$ after creation of wounds. TPA increased the motility of non-invasive MCF-7 cells, but pretreatment of cells with conditioned media containing various hardness of DSW from 400-1,500 resulted in decreased ability of the cells to close the wound in the wound healing assay. (B) Effects of DSW on regulation of MMP-9 activity in a gelatin zymography assay. The collected media from cells treated with DSW were electrophoresed in an $8 \%$ sodium dodecyl sulfate (SDS)-polyacrylamide gel containing $0.1 \%$ (v/v) gelatin. The relative enzyme activity of each sample was expressed as fold change compared to control. (C) Effects of DSW on the expression of MMP-9. Total cellular mRNA was isolated from cells treated with different hardness of DSW in the presence of TPA, and RT-PCR was conducted to measure the expression of MMP-9. The relative intensity of each sample was expressed as fold change compared to control after normalization to a respective loading control.

containing 800 or 1,500 hardness DSW was about $30 \%$ less than the TPA-induced MMP-9 activity (Fig. 4B).

Inhibitory effect of DSW on MMP-9 activity is via suppressed MMP-9 gene expression rather than $u P A / u P A R$ system. To understand the mechanism underlying the suppression of MMP-9 activity by DSW, we first examined whether treatment with DSW directly affects the gene expression of MMP-9. Cells were treated with different hardness of DSW. After $2 \mathrm{~h}$, cells were treated with TPA, and then continuously cultured for another $24 \mathrm{~h}$ before isolating the total cellular mRNA for RT-PCR. As shown in Fig. 4C, gene expression of MMP-9 was induced in cells treated with TPA. However, treatment with DSW clearly inhibited MMP-9 gene expression (Fig. 4C). It is well known that the activation of MMP-9 is mediated by active uPA, which catalyzes the cleavage of plasminogen to generate active plasmin. In turn, plasmin facilitates the release of several proteolytic enzymes, including MMPs. Thus, we tested 
A
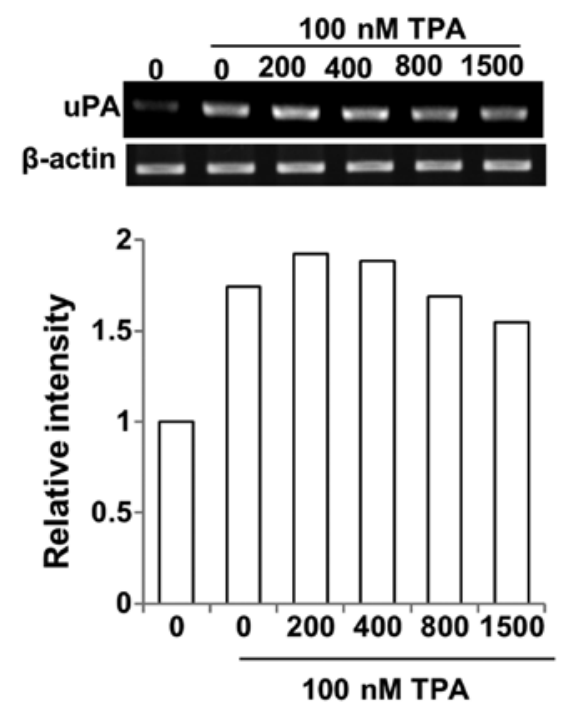

$100 \mathrm{nM}$ TPA

B
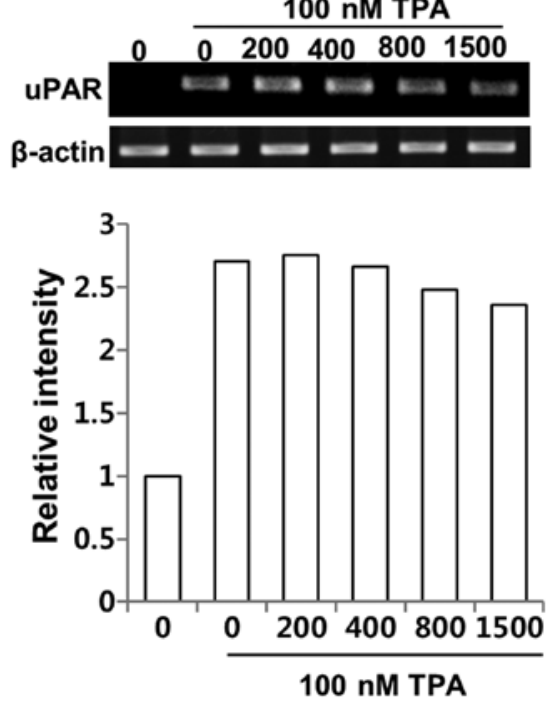

Figure 5. Effects of DSW on uPA/uPAR mRNA expression in MCF-7 cells The mRNA expression of (A) UPA and (B) UPAR were analyzed by RT-PCR. MCF-7 cells were treated with conditioned media containing various hardness of DSW for $2 \mathrm{~h}$ prior to addition of $100 \mathrm{nM}$ TPA. Cells were further incubated for $24 \mathrm{~h}$ and harvested for RNA isolation. The relative intensity of each sample was expressed as fold change compared to control after normalization to a respective loading control.

whether the inhibitory effects of DSW on MMP-9 activity result from suppressed expression of UPA and its receptor, UPAR, in DSW-treated MCF-7 cells. The effect of TPA in inducing UPA and UPAR expression was significant, but DSW exhibited only mild inhibitory effects on uPA and uPAR expression (Fig. 5A and B). Taken together, our data suggest that DSW inhibits TPA-induced MMP-9 activity mainly through the inhibition of MMP-9 gene expression rather than by inhibition of uPA/ uPAR expression.

Regulation of the expression of TGF- $\beta$ and Wnt ligands by $D S W$ in MCF-7 cells. We evaluated the effects of DSW on the mRNA expression of TGF- $\beta$. The control cells showed barely detectable mRNA expression of TGF- $\beta$, while treatment with TPA for $24 \mathrm{~h}$ showed significant induction of TGF- $\beta$ expression. However, treatment with DSW inhibited the TGF- $\beta$ gene expression of MCF-7 cells; in particular, conditioned media containing 800 or 1,500 hardness DSW inhibited the TPA-induced TGF- $\beta$ expression by approximately $60 \%$ (Fig. 6A). The expression of Wnt5a was also affected by treatment with DSW (Fig. 6B). Similar to the mRNA expression of TGF- $\beta$, the endogenous expression of Wnt5a was very low, but treatment with TPA efficiently induced the expression of Wnt5a. Inhibitory effect of DSW on the TPA-induced Wnt5a expression was significant thereby exhibiting over 50\% inhibition in cells treated with 800 or 1,500 hardness DSW (Fig. 6B). We further investigated whether DSW can alter the expression of the Wnt-canonical signaling ligand, Wnt3a. Its binding to Wnt receptors such as FZD and LRP5/6 promoted stabilization and nuclear translocation of $\beta$-catenin, resulting in the activation of target genes with cancer-promoting roles (17). TPA mildly induced the expression of Wnt3a, but DSW efficiently inhibited TPA-induced Wnt3a expression (Fig. 6C), implying that DSW may also inhibit the Wnt signaling-involved tumor migration and metastasis.

\section{Discussion}

In this study, we have evaluated the potential health benefit of DSW on the inhibition of metastatic potential of human breast cancer cells. We showed that treatment with DSW efficiently inhibited the migratory ability of highly invasive MDA-MB-231 cells in a wound healing assay. This effect of DSW appears to be mediated through TGF- $\beta$ and Wnt5a signaling, subsequently resulting in the attenuated expression of CD44. TGF- $\beta$ is one of the well known signaling pathways that are involved in the survival of breast cancer cells and cell migration (32). Moreover, abrogation of autocrine TGF- $\beta$ signaling in MDA-MB-231 cells with transfection of a kinase-inactive type II TGF- $\beta$ receptor impaired the basal migratory potential by blocking both Smad-dependent and -independent signaling pathways (33). The inhibitory effect of DSW on the Wnt5a gene expression was relatively mild compared to its effect on TGF- $\beta$ gene expression. Since TGF- $\beta$ directly upregulates Wnt5a expression through the Smad complex, the mild inhibitory effects of DSW on Wnt5a gene expression may indicate that the relative contribution of Smaddependent TGF- $\beta$ signaling is moderate in mediating motility compared to that of Smad-independent TGF- $\beta$ signaling. The inhibitory effects of DSW on Smad-independent TGF- $\beta$ signaling need to be clarified in future studies.

CD44 is also known to be involved in Wnt5a-dependent invasion and metastasis. CD44 is a transmembrane glycoprotein that participates in many cellular processes including regulation of cell-cell interactions, migration and adhesion. It is aberrantly expressed in breast cancers and has been implicated in the metastatic process as well as in the putative cancer stem cell compartment (24). In this study, we showed that treatment with DSW attenuated the overall expression of CD44, suggesting that the inhibitory effect of DSW on Wnt5a expression subsequently affected the expression of CD44 in MDA-MB-231 cells. Besides CD44, the expression of MMPs is also regulated by Wnt5a via binding to Ror2 (25). 

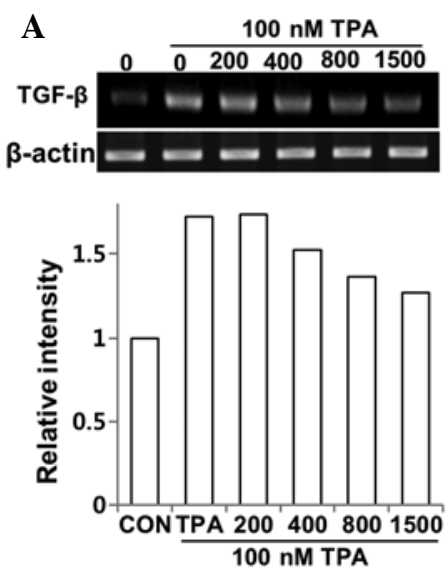
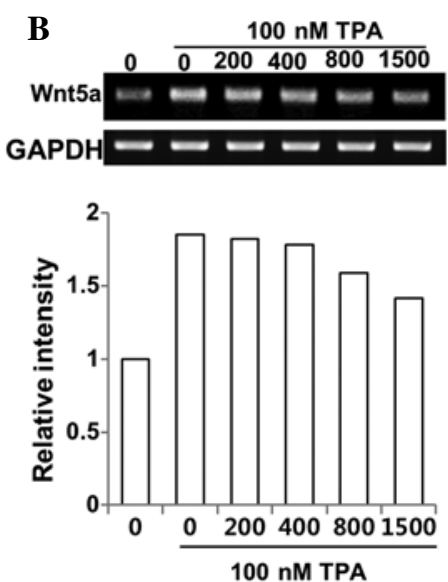
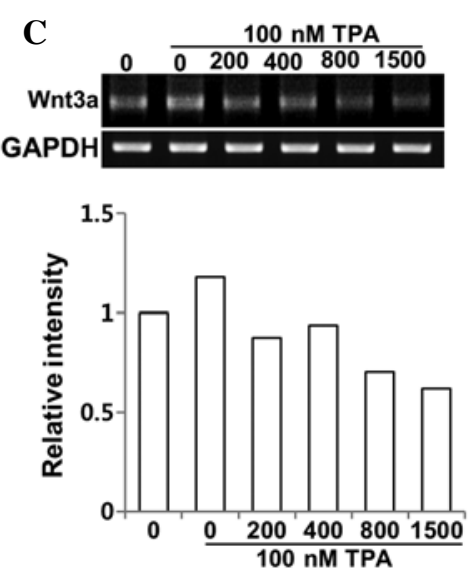

Figure 6. Secretion of Wnt ligands and TGF- $\beta$ from TPA-treated MCF-7 cells was inhibited by DSW. The mRNA expression of (A) TGF- $\beta$, (B) Wnt5a and (c) Wnt3a were analyzed by RT-PCR. MCF-7 cells were treated with conditioned media containing various hardness of DSW for $2 \mathrm{~h}$ prior to addition of $100 \mathrm{nM}$ TPA. Cells were further incubated for $24 \mathrm{~h}$ and harvested for RNA isolation. The relative intensity of each sample was expressed as fold change compared to control after normalization to a respective loading control.

We further investigated the preventive effect of DSW on TPA-induced invasive/metastatic tumor features in non-invasive MCF-7 cells. Similar to the inhibitory effects shown in MDA-MB-231 cells, DSW treatment inhibited TPA-induced migration and MMP-9 activity with a concomitant decrease in mRNA levels of MMP-9, TGF- $\beta$ and Wnt5a. Interestingly, we observed that DSW can alter the expression of Wnt-canonical signaling ligand, Wnt3a. Aberrant activation of Wnt signaling was observed in a variety of cancers, implying the importance of Wnt signaling in carcinogenesis (34). Wnt signaling also mediates the metastatic progression by enhancing the migratory ability of cancer cells. On top of that, blockade of Wnt signaling reduces motility and lung metastasis as well as tumor outgrowth, suggesting the involvement of Wnt signaling in tumor invasion and migration (35). Thus, the inhibitory effect of DSW on Wnt3a expression implies that the Wnt signaling pathway mediating the cell migration and invasion may be affected by treatment with DSW.

Although it is not clear which component of DSW affects the metastatic potential of human breast cancer cells, it is presumed that the combined ionic action of several minerals such as calcium, magnesium and zinc, may play important roles in mediating the inhibition of metastasis. Among these, calcium and magnesium are the chief mineral ions present in DSW. The concentration of calcium is approximately $100 \mathrm{mg} / 1$ in 1,500 hardness DSW, while the amount of magnesium is approximately $300 \mathrm{mg} / 1$.

Calcium, a key cellular mediator, has been implicated in the induction of apoptosis and regulation of apoptosis signaling pathway in human breast cancer cells via the activation of $\mathrm{Ca}^{2+}$-dependent proapoptotic protease calpain and caspase-12 (36). Epidemiologic studies also suggest that intake of calcium appear to reduce the risk of breast cancer $(37,38)$, as well as colon cancer $(7,10)$. Individuals with a calcium intake of more than $700 \mathrm{mg}$ per day had a 35 to $45 \%$ reduced risk of cancer of the distal part of the colon than those who had a calcium intake of $500 \mathrm{mg}$ or less per day. However, there was no incremental benefit of additional calcium intake beyond $700 \mathrm{mg}$ / day (10). Furthermore, improving calcium and vitamin D nutritional status substantially reduced all-cancer risk in postmenopausal women (7). Although some studies do not support a benefit of calcium $(39,40)$, the majority of studies suggest calcium supplements with vitamin D have shown some promise in cancer risk reduction.

Magnesium deficiency is also known to be associated with advancement of malignancy and metastasis. Decreased serum levels of magnesium are frequently observed in patients with tumors $(6,9)$, and low magnesium status was observed in cancer patients undergoing several courses of chemotherapy (41). In contrast, daily intake of an additional $100 \mathrm{mg}$ of magnesium reduced the risk of colorectal cancer by $12 \%$ (42), suggesting beneficial effects of magnesium against multiple cancers. Nevertheless, the deficiency of extracellular magnesium ceased tumor cell growth, its deficiency caused development of more lung metastasis than controls despite smaller size of the primary tumor in mice (8).

The exact mechanism underlying metastasis with regard to magnesium deficiency is unclear, but a gene expression array of tumors from magnesium deficient mice suggested that magnesium influences the remodeling of extracellular matrix via upregulation of several proteases such as metalloproteinase, calpain and others (43). Therefore, the inhibitory effects of DSW on migration and MMP-9 activity may have occurred from a high concentration of magnesium within DSW. Nevertheless, mineral water containing only exogenous magnesium as 1,500 hardness did not show significant inhibitory effects against metastatic potential of human breast cancer cells compared to effects of DSW (data not shown). Thus, the presence of the proper ratio of magnesium to calcium in DSW may provide synergetic effects via the inter-relationship between magnesium and calcium. In this regard, recent studies have pointed out the importance of the ratio of calcium to magnesium, which can modify their effects on carcinogenesis (44). Both high magnesium and calcium levels have been linked to reduced risks of cancer, but studies have also shown that high calcium levels inhibit the absorption of magnesium. In fact, the results from a large clinical trial found that supplementation of calcium reduced the risk 
of cancer recurrence only if the ratio of calcium to magnesium was low, suggesting a need for both minerals in reducing the risk of cancer (44). Thus, DSW may be a beneficial source that provides the proper ratio of calcium to magnesium.

Our data showed substantial inhibitory effects of DSW on the metastatic potential of human breast cancer cells. The mechanism underlying DSW-mediated suppression on tumor migration and invasion were not fully elucidated in this study, but we believe that this is the first study to provide an important clue of a possible mechanism for the inhibitory effects of DSW on the Wnt signaling pathway, which mediates cell migration and invasion in coordination with the TGF- $\beta$ signaling pathway. Taken together, our data showed that DSW has inhibitory effects on breast cancer invasion/metastasis, suggesting that DSW shows some promise in improving cancer survival by preventing tumor metastasis.

\section{Acknowledgements}

This study was supported by the project entitled 'Development of Technology for support of deep-sea water industry (PJT200014)' from the Ministry of Land, Transport and Maritime Affairs, Korea.

\section{References}

1. Nakasone T and Akeda S: The application of deep sea water in Japan. UJNR Technical Report 28: 69-75, 1999.

2. Shon YH, Kim JS and Nam KS: Inhibitory effect of Deep-sea water on cytochrome P450 1A1, aromatase, and MMP-9. J Life Sci 18: 503-508, 2008.

3. Hwang HS, Kim SH, Yoo YG, et al: Inhibitory effect of deep-sea water on differentiation of 3T3-L1 adipocytes. Mar Biotechnol (NY) 11: 161-168, 2009.

4. Radhakrishnan G, Yamamoto M, Maeda H, et al: Intake of dissolved organic matter from deep seawater inhibits atherosclerosis progression. Biochem Biophys Res Commun 387: 25-30, 2009.

5. Lee KS, Shin JS, Kwon YS, Moon DS and Nam KS: Suppression of cancer progression and metastasis in HT-29 human colorectal adenocarcinoma by deep sea water. Biotechnol Bioproc Eng 18: 194-200, 2013

6. Kohli GS, Bhargava A, Goel H, et al: Serum magnesium levels in patients with head and neck cancer. Magnesium 8: 77-86, 1989.

7. Lappe JM, Travers-Gustafson D, Davies KM, Recker RR and Heaney RP: Vitamin D and calcium supplementation reduces cancer risk: results of a randomized trial. Am J Clin Nutr 85: 1586-1591, 2007.

8. Nasulewicz A, Wietrzyk J, Wolf FI, et al: Magnesium deficiency inhibits primary tumor growth but favors metastasis in mice. Biochim Biophys Acta 1739: 26-32, 2004.

9. Sartori S, Nielsen I, Tassinari D, et al: Serum and erythrocyte magnesium concentrations in solid tumours: relationship with stage of malignancy. Magnes Res 5: 189-192, 1992.

10. Wu K, Willett WC, Fuchs CS, Colditz GA and Giovannucci EL: Calcium intake and risk of colon cancer in women and men. J Natl Cancer Inst 94: 437-446, 2002.

11. Gamucci T, D'Ottavio AM, Magnolfi E, et al: Weekly epirubicin plus docetaxel as first-line treatment in metastatic breast cancer. Br J Cancer 97: 1040-1045, 2007.

12. Padua D and Massague J: Roles of TGFbeta in metastasis. Cell Res 19: 89-102, 2009.

13. Shariat SF, Shalev M, Menesses-Diaz A, et al: Preoperative plasma levels of transforming growth factor beta(1) (TGFbeta(1)) strongly predict progression in patients undergoing radical prostatectomy. J Clin Oncol 19: 2856-2864, 2001.

14. Tsushima $\mathrm{H}$, Ito $\mathrm{N}$, Tamura $\mathrm{S}$, et al: Circulating transforming growth factor beta 1 as a predictor of liver metastasis after resection in colorectal cancer. Clin Cancer Res 7: 1258-1262, 2001.
15. Yang YA, Dukhanina O, Tang B, et al: Lifetime exposure to a soluble TGF-beta antagonist protects mice against metastasis without adverse side effects. J Clin Invest 109: 1607-1615, 2002.

16. Yin JJ, Selander K, Chirgwin JM, et al: TGF-beta signaling blockade inhibits PTHrP secretion by breast cancer cells and bone metastases development. J Clin Invest 103: 197-206, 1999.

17. Howe LR and Brown AM: Wnt signaling and breast cancer. Cancer Biol Ther 3: 36-41, 2004.

18. Katoh M: Transcriptional mechanisms of WNT5A based on NF-kappaB, Hedgehog, TGFbeta, and Notch signaling cascades. Int J Mol Med 23: 763-769, 2009.

19. Lejeune S, Huguet EL, Hamby A, Poulsom R and Harris AL: Wnt5a cloning, expression, and up-regulation in human primary breast cancers. Clin Cancer Res 1: 215-222, 1995.

20. Smith K, Bui TD, Poulsom R, Kaklamanis L, Williams G and Harris AL: Up-regulation of macrophage wnt gene expression in adenoma-carcinoma progression of human colorectal cancer. Br J Cancer 81: 496-502, 1999.

21. Bittner M, Meltzer P, Chen Y, et al: Molecular classification of cutaneous malignant melanoma by gene expression profiling. Nature 406: 536-540, 2000.

22. Weeraratna AT, Jiang Y, Hostetter G, et al: Wnt5a signaling directly affects cell motility and invasion of metastatic melanoma. Cancer Cell 1: 279-288, 2002.

23. Dissanayake SK, Wade M, Johnson CE, et al: The Wnt5A/ protein kinase $\mathrm{C}$ pathway mediates motility in melanoma cells via the inhibition of metastasis suppressors and initiation of an epithelial to mesenchymal transition. J Biol Chem 282: 17259-17271, 2007.

24. Dietrich A, Tanczos E, Vanscheidt W, Schopf E and Simon JC: High CD44 surface expression on primary tumours of malignant melanoma correlates with increased metastatic risk and reduced survival. Eur J Cancer 33: 926-930, 1997.

25. O'Connell MP, Fiori JL, Xu M, et al: The orphan tyrosine kinase receptor, ROR2, mediates Wnt5A signaling in metastatic melanoma. Oncogene 29: 34-44, 2010.

26. Nabeshima K, Inoue T, Shimao Y and Sameshima T: Matrix metalloproteinases in tumor invasion: role for cell migration. Pathol Int 52: 255-264, 2002.

27. Itoh T, Tanioka M, Yoshida H, Yoshioka T, Nishimoto $H$ and Itohara S: Reduced angiogenesis and tumor progression in gelatinase A-deficient mice. Cancer Res 58: 1048-1051, 1998.

28. Perou CM, Sorlie T, Eisen MB, et al: Molecular portraits of human breast tumours. Nature 406: 747-752, 2000.

29. Subramaniam DS and Isaacs C: Utilizing prognostic and predictive factors in breast cancer. Curr Treat Options Oncol 6: 147-159, 2005.

30. Klemm F, Bleckmann A, Siam L, et al: beta-catenin-independent WNT signaling in basal-like breast cancer and brain metastasis. Carcinogenesis 32: 434-442, 2011.

31. Kao J, Salari K, Bocanegra M, et al: Molecular profiling of breast cancer cell lines defines relevant tumor models and provides a resource for cancer gene discovery. PLoS One 4: e6146, 2009.

32. Lei X, Yang J, Nichols RW and Sun LZ: Abrogation of TGFbeta signaling induces apoptosis through the modulation of MAP kinase pathways in breast cancer cells. Exp Cell Res 313: 1687-1695, 2007.

33. Dumont N, Bakin AV and Arteaga CL: Autocrine transforming growth factor-beta signaling mediates Smad-independent motility in human cancer cells. J Biol Chem 278: 3275-3285, 2003.

34. Anastas JN and Moon RT: WNT signalling pathways as therapeutic targets in cancer. Nat Rev Cancer 13: 11-26, 2013.

35. Matsuda Y, Schlange T, Oakeley EJ, Boulay A and Hynes NE: WNT signaling enhances breast cancer cell motility and blockade of the WNT pathway by sFRP1 suppresses MDA-MB-231 xenograft growth. Breast Cancer Res 11: R32, 2009.

36. Mathiasen IS, Sergeev IN, Bastholm L, Elling F, Norman AW and Jaattela M: Calcium and calpain as key mediators of apoptosis-like death induced by vitamin D compounds in breast cancer cells. J Biol Chem 277: 30738-30745, 2002.

37. Cui Y and Rohan TE: Vitamin D, calcium, and breast cancer risk: a review. Cancer Epidemiol Biomarkers Prev 15: 1427-1437, 2006.

38. Shin MH, Holmes MD, Hankinson SE, Wu K, Colditz GA and Willett WC: Intake of dairy products, calcium, and vitamin d and risk of breast cancer. J Natl Cancer Inst 94: 1301-1311, 2002 . 
39. Benito E, Stiggelbout A, Bosch FX, et al: Nutritional factors in colorectal cancer risk: a case-control study in Majorca. Int $\mathrm{J}$ Cancer 49: 161-167, 1991

40. Negri E, La Vecchia C, D'Avanzo B and Franceschi S: Calcium, dairy products, and colorectal cancer. Nutr Cancer 13: 255-262, 1990.

41. Lajer H and Daugaard G: Cisplatin and hypomagnesemia. Cancer Treat Rev 25: 47-58, 1999.

42. Wark PA, Lau R, Norat T and Kampman E: Magnesium intake and colorectal tumor risk: a case-control study and metaanalysis. Am J Clin Nutr 96: 622-631, 2012.

43. Maier JA, Nasulewicz-Goldeman A, Simonacci M, Boninsegna A, Mazur A and Wolf FI: Insights into the mechanisms involved in magnesium-dependent inhibition of primary tumor growth. Nutr Cancer 59: 192-198, 2007.

44. Dai Q, Shrubsole MJ, Ness RM, et al: The relation of magnesium and calcium intakes and a genetic polymorphism in the magnesium transporter to colorectal neoplasia risk. Am J Clin Nutr 86: 743-751, 2007.
45. Jang JY, Jeon YK and Kim CW: Degradation of HER2/neu by ANT2 shRNA suppresses migration and invasiveness of breast cancer cells. BMC Cancer 10: 391, 2010.

46. Kang JH, Song KH, Jeong KC, et al: Involvement of Cox-2 in the metastatic potential of chemotherapy-resistant breast cancer cells. BMC Cancer 11: 334, 2011.

47. Li Y and Sarkar FH: Gene expression profiles of genisteintreated PC3 prostate cancer cells. J Nutr 132: 3623-3631, 2002.

48. Suzuki A, Ozono K, Kubota T, Kondou H, Tachikawa K and Michigami T: PTH/cAMP/PKA signaling facilitates canonical Wnt signaling via inactivation of glycogen synthase kinase3beta in osteoblastic Saos-2 cells. J Cell Biochem 104: 304-317, 2008.

49. Takeuchi E, Tanaka T, Umemoto E, et al: VLA-4-dependent and -independent pathways in cell contact-induced proinflammatory cytokine production by synovial nurse-like cells from rheumatoid arthritis patients. Arthritis Res 4: R10, 2002. 